\title{
Lifetime measurements using two-step laser excitation for high-lying even-parity levels and improved theoretical oscillator strengths in Y II
}

\author{
P. Palmeri, ${ }^{1 \star}$ P. Quinet,${ }^{1,2}$ H. Lundberg, ${ }^{3}$ L. Engström, ${ }^{3}$ H. Nilsson ${ }^{4}$ \\ and H. Hartman ${ }^{4,5}$ \\ ${ }^{1}$ Physique Atomique et Astrophysique, Université de Mons - UMONS, 20 Place du Parc, B-7000 Mons, Belgium \\ ${ }^{2}$ IPNAS, Université de Liège, Campus du Sart-Tilman, B-4000 Liège, Belgium \\ ${ }^{3}$ Department of Physics, Lund University, Box 118, SE-221 00 Lund, Sweden \\ ${ }^{4}$ Lund Observatory, Lund University, Box 43, SE-221 00 Lund, Sweden \\ ${ }^{5}$ Material Sciences and Applied Mathematics, Malmö University, SE-205 06 Malmö, Sweden
}

Accepted 2017 June 23. Received 2017 June 23; in original form 2017 March 16

\begin{abstract}
We report new time-resolved laser-induced fluorescence lifetime measurements for 22 highly excited even-parity levels in singly ionized yttrium (Y II). To populate these levels belonging to the configurations $4 \mathrm{~d} 6 \mathrm{~s}, 5 \mathrm{~s} 6 \mathrm{~s} 4 \mathrm{~d} 5 \mathrm{~d}, 5 \mathrm{p}^{2}, 4 \mathrm{~d} 7 \mathrm{~s}$ and $4 \mathrm{~d} 6 \mathrm{~d}$, a two-step laser excitation technique was used. Our previous pseudo-relativistic Hartree-Fock model (Biémont et al. 2011) was improved by extending the configuration interaction up to $n=10$ to reproduce the new experimental lifetimes. A set of semi-empirical oscillator strengths extended to transitions falling in the spectral range $\lambda \lambda 194-3995 \mathrm{~nm}$, depopulating these 22 even-parity levels in Y II, is presented and compared to the values found in the Kurucz's data base (Kurucz 2011).
\end{abstract}

Key words: atomic data-atomic processes - methods: numerical.

\section{INTRODUCTION}

Accurate oscillator strengths for electric dipole transitions in $Y_{\text {II }}$ are needed for the determination of the yttrium abundance in stellar atmospheres. A recent example is the determination of the abundance ratio $[\mathrm{Y} / \mathrm{Mg}]$ in solar twins that provides a sensitive chronometer for Galactic evolution (Nissen 2015; Tucci Maia et al. 2016). Yttrium $(Z=39)$ is a slow neutron-capture element primarily produced in low-to-medium mass AGB stars at solar metallicity, and its presence in stars of different ages and locations gives a good indication of the chemical history of the Milky Way (Mishenina et al. 2016).

High-excitation lines have additional diagnostic value because they can probe both non-local thermodynamical equilibrium and 3D effects in stellar atmospheres (Lind, Bergeman \& Asplund 2012). It is worth noting that all previous experimental lifetimes and oscillator strengths available in the literature for $\mathrm{Y}$ II only involve low-excited odd-parity levels (Andersen, Ramanujan \& Bahr 1978; Hannaford et al. 1982; Gorshklov \& Komarovskii 1986; Pitts \& Newson 1986; Wännström et al. 1988; Reshetnikova \& Skorokhod 1999; Biémont et al. 2011). With the exception of the Kurucz's data base (Kurucz 2011), this is also the case for the theoretical data (Pirronello \& Strazzulla 1980; Migdalek \& Baylis 1987; Migdalek \& Stanek 1993; Biémont et al. 2011). Hannaford et al. (1982) combine the experimental lifetimes with relative intensities of the lines depopulating these levels to derive oscillator strengths.

^E-mail: patrick.palmeri@umons.ac.be
The aim of this study is to extend our knowledge of Y II to include highly excited even-parity levels. This was accomplished with a two-step laser excitation technique at the Lund High Power Laser Facility VUV laboratory using time-resolved laser-induced fluorescence (TR-LIF). Our previous HFR+CPOL calculations (Biémont et al. 2011) have been extended up to $n=10$ to provide the radiative rates for the transitions depopulating the whole set of measured odd-parity and even-parity levels.

In Section 2, a description of the experimental method is given. Section 3 describes our new HFR+CPOL calculations. The results are presented and discussed in Section 4.

\section{TR-LIF MEASUREMENTS}

The ground state in $\mathrm{Y}_{\text {II }}$ is $5 \mathrm{~s}^{2}{ }^{1} \mathrm{~S}_{0}$ and the lowest excited term is $4 \mathrm{~d} 5 \mathrm{~s}{ }^{3} \mathrm{D}$, with levels below $1500 \mathrm{~cm}^{-1}$. These even-parity levels are directly populated in the ablation plasma created by focusing a frequency doubled Nd:YAG laser on a rotating yttrium target inside a vacuum chamber with a pressure of about $10^{-4} \mathrm{mbar}$. To reach the highly excited even-parity levels, we applied a two-step procedure. A Nd:YAG pumped dye laser, with a pulse length of around $10 \mathrm{~ns}$ and operating on a Pyridin dye, excited the intermediate odd-parity levels in the 4d5p configuration around $29000 \mathrm{~cm}^{-1}$. A second $\mathrm{Nd}$ :YAG pumped dye laser, with a pulse length of $0.8 \mathrm{~ns}$ and operating on DCM dye, excited the final, even-parity levels, in the energy range $50000-75000 \mathrm{~cm}^{-1}$ studied in this investigation. An 


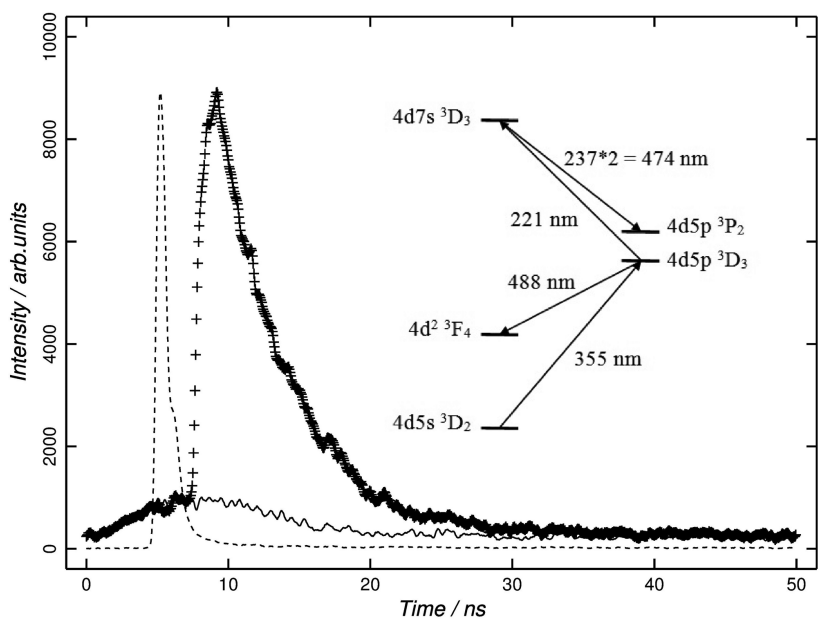

Figure 1. The + signs show the measured decay of the $4 \mathrm{~d} 7 \mathrm{~s}{ }^{3} \mathrm{D}_{3}$ level in $\mathrm{Y}_{\mathrm{II}}$ in the second spectral order at $474 \mathrm{~nm}$, perturbed by the first-order decay from the intermediate $4 \mathrm{~d} 5 \mathrm{p}^{3} \mathrm{D}_{3}$ level at $488 \mathrm{~nm}$. The insert illustrates schematically the excitation and decay channels involved. The lower curve (solid line) is a separate measurement at $474 \mathrm{~nm}$ with the second-step laser blocked, revealing the perturbation. This curve is then subtracted from the actual decay measurement. The dashed curve shows the second-step excitation laser, with a full width at half-maximum of $0.8 \mathrm{~ns}$ and displaced in time for clarity.

example of the two-step procedure is shown schematically in the insert in Fig. 1. Details of the set-up at the high-power laser facility in Lund are given by Lundberg et al. (2016), and an overview is presented in fig. 1 of that paper.
To reach the $4 \mathrm{~d} 5 \mathrm{p}$ levels, wavelengths around $350 \mathrm{~nm}$ were obtained by frequency doubling of the dye-laser output in a KDP crystal. For the final step, we utilized frequency doubling or tripling and, when necessary, added or subtracted one Stokes shift of $4153 \mathrm{~cm}^{-1}$ in a $\mathrm{H}_{2}$ gas cell. The fluorescence from the excited levels was detected by a $1 / 8 \mathrm{~m}$ monochromator, with its $0.12 \mathrm{~mm}$ wide entrance slit oriented parallel to the excitation laser beams and perpendicular to the ablation laser, and registered by a fast micro-channel-plate PM-tube (Hamamatsu R 3809U) with a rise time of 200 ps. A Tektronix oscilloscope (DPO 7254) digitized both the fluorescence signal and the shape of the second-step excitation laser, recorded by a fast photodiode, in time steps of $50 \mathrm{ps}$. The different excitation and detection schemes used are presented in Table 1.

Each recorded decay curve was averaged over 1000 laser shots, and for each level we performed between 10 and 20 measurements over several days. All curves were analysed by fitting a single exponential decay convoluted by the recorded laser pulse and a constant background using the code DECFIT (Palmeri et al. 2008). The final lifetime is the average over all measurements, and is presented in Table 2. The quoted uncertainties include statistical uncertainties from the curve fitting and the variation between the repeated measurements, where the latter is the dominating source.

As discussed by Lundberg et al. (2016), there are two special experimental considerations in a two-step scheme. A problem may arise if there is a decay channel from the intermediate level close in wavelength to the channel used to measure the decay of the final level. Since the intermediate fluorescence is usually very intense and extends over more than $10 \mathrm{~ns}$, this may cause problems even with a fairly large wavelength separation. One such case is illustrated in Fig. 1. Here, the transition at $488 \mathrm{~nm}$ from the intermediate

Table 1. Two-step excitation schemes in Y II.

\begin{tabular}{|c|c|c|c|c|c|c|c|}
\hline \multirow[b]{2}{*}{ Final level } & \multicolumn{3}{|c|}{ First-step excitation $^{a}$} & \multicolumn{3}{|c|}{ Second-step excitation } & \multirow{2}{*}{$\begin{array}{c}\text { Detection } \\
\lambda_{\text {air }}^{d} \\
(\mathrm{~nm})\end{array}$} \\
\hline & $\begin{array}{l}\text { Start level }{ }^{b} \\
\left(\mathrm{~cm}^{-1}\right)\end{array}$ & $\begin{array}{l}\text { Intermediate }^{b} \\
\quad\left(\mathrm{~cm}^{-1}\right)\end{array}$ & $\begin{array}{c}\lambda_{\text {air }} \\
(\mathrm{nm})\end{array}$ & $\begin{array}{l}\text { Final level }^{b} \\
\quad\left(\mathrm{~cm}^{-1}\right)\end{array}$ & $\begin{array}{l}\lambda_{\text {air }} \\
(\mathrm{nm})\end{array}$ & Scheme $^{c}$ & \\
\hline $4 d 6 \mathrm{se}^{3} \mathrm{D}_{1}$ & 1045 & 28730 & 361.12 & 54956.08 & 381.19 & $2 \omega+S$ & 347 \\
\hline $4 d 6 \mathrm{se}^{3} \mathrm{D}_{2}$ & 1045 & 28730 & 361.12 & 55032.35 & 380.09 & $2 \omega+S$ & $346^{e}, 461$ \\
\hline $4 d 6 \mathrm{~s} \mathrm{e}^{3} \mathrm{D}_{3}$ & 1045 & 28730 & 361.12 & 55645.64 & 371.43 & $2 \omega+S$ & 428 \\
\hline $4 d 6 s e^{1} D_{2}$ & 0 & 27516 & 363.31 & 55725.52 & 354.40 & $2 \omega+S$ & $338^{e}, 446$ \\
\hline $5 s 6 \mathrm{~s} \mathrm{e}^{3} \mathrm{~S}_{1}$ & 0 & 27516 & 363.31 & 58263.24 & 325.15 & $2 \omega$ & 297,384 \\
\hline $4 d 5 d e^{1} F_{3}$ & 1449 & 28394 & 371.02 & 58533.30 & 331.70 & $2 \omega$ & 309 \\
\hline $4 d 5 d^{3} D_{1}$ & 1045 & 28595 & 362.87 & 58720.38 & 331.85 & $2 \omega$ & $307,375^{e}$ \\
\hline $5 \mathrm{p}^{2} \mathrm{e}^{3} \mathrm{P}_{0}$ & 1045 & 28595 & 362.87 & 58776.42 & 331.23 & $2 \omega$ & 286 \\
\hline $4 d 5 d^{3} D_{2}$ & 1045 & 28595 & 362.87 & 58947.62 & 329.37 & $2 \omega$ & $318,373^{e}$ \\
\hline $5 \mathrm{p} 2 \mathrm{e}^{3} \mathrm{P}_{1}$ & 1045 & 28730 & 361.12 & 59147.56 & 328.66 & $2 \omega$ & $280,283,290$ \\
\hline $4 d 5 d^{3} \mathrm{e}_{3}$ & 1045 & 29214 & 354.91 & 59179.59 & 333.62 & $2 \omega$ & 303,313 \\
\hline $4 d 5 \mathrm{df}^{3} \mathrm{D}_{3}$ & 1045 & 29214 & 354.91 & 59327.89 & 331.98 & $2 \omega$ & 311,314 \\
\hline $4 d 5 d^{3} \mathrm{G}_{4}$ & 1045 & 29214 & 354.91 & 59472.65 & 330.39 & $2 \omega$ & 313 \\
\hline $5 \mathrm{p}^{2} \mathrm{e}^{3} \mathrm{P}_{2}$ & 1045 & 29214 & 354.91 & 59670.26 & 328.24 & $2 \omega$ & 278,285 \\
\hline $4 d 5 d e^{1} P_{1}$ & 1045 & 28595 & 362.87 & 59716.84 & 321.23 & $2 \omega$ & $298,308,310$ \\
\hline $4 d 5 d e^{3} G_{5}$ & 1449 & 28394 & 371.02 & 59900.52 & 317.30 & $2 \omega$ & 317 \\
\hline $5 \mathrm{p}^{2} \mathrm{f}^{1} \mathrm{D}_{2}$ & 1045 & 28730 & 361.12 & 60535.92 & 314.32 & $2 \omega$ & 278,303 \\
\hline $4 d 5 d f^{3} P_{1}$ & 1045 & 28730 & 361.12 & 64263.74 & 281.34 & $2 \omega+\mathrm{AS}$ & 281,312 \\
\hline $4 d 5 \mathrm{df}^{3} \mathrm{P}_{2}$ & 1045 & 29214 & 354.91 & 64597.24 & 282.54 & $2 \omega+\mathrm{AS}$ & 282,309 \\
\hline $4 \mathrm{~d} 7 \mathrm{~s}{ }^{3} \mathrm{D}_{3}$ & 1045 & 29214 & 354.91 & 74374.91 & 221.36 & $3 \omega$ & $237^{e}$ \\
\hline \multirow[t]{2}{*}{$4 d 7 s{ }^{1} D_{2}$} & 1045 & 29214 & 354.91 & 74582.56 & 220.35 & $3 \omega$ & 242 \\
\hline & 1045 & 28730 & 361.12 & & 218.02 & $3 \omega$ & 242 \\
\hline $4 d 6 d^{3} D_{3}$ & 1045 & 29214 & 354.91 & 76178.28 & 212.86 & $3 \omega$ & 228 \\
\hline
\end{tabular}

Notes. ${ }^{a}$ For all measured levels, the first excitation step used the frequency doubled $(2 \omega)$ output from the dye laser.

${ }^{b}$ Nilsson et al (1991).

${ }^{c} 2 \omega / 3 \omega$ means the frequency doubled/tripled output from the dye laser. S/AS is one added/subtracted Stokes shift of $4153 \mathrm{~cm}^{-1}$.

${ }^{d}$ Fluorescence measurements below $400 \mathrm{~nm}$ were performed in the second spectral order.

${ }^{e}$ Corrected for fluorescence from the level excited by the first-step laser. See Fig. 1 and discussion in the text for further details. 
P. Palmeri et al.

Table 2. Comparison between experimental and theoretical lifetimes of selected levels in Y II.

\begin{tabular}{|c|c|c|c|c|c|}
\hline \multirow[t]{2}{*}{ Level $^{a}$} & \multirow{2}{*}{$\begin{array}{l}\text { Energy }^{a} \\
\left(\mathrm{~cm}^{-1}\right)\end{array}$} & \multicolumn{2}{|c|}{ Experimental lifetime (ns) } & \multicolumn{2}{|c|}{ Theoretical lifetime (ns) } \\
\hline & & This work ${ }^{b}$ & Others & This work $^{c}$ & Others \\
\hline $5 \mathrm{~s} 5 \mathrm{pz}^{3} \mathrm{P}_{0}^{\mathrm{o}}$ & 23445.063 & & $54.7 \pm 1.0^{e}$ & 64.48 & $\begin{array}{l}31.88^{h} \\
38.31^{i}\end{array}$ \\
\hline $5 \mathrm{~s} 5 \mathrm{pz}^{3} \mathrm{P}_{1}^{\mathrm{o}}$ & 23776.245 & & $51.5 \pm 1.0^{e}$ & $57.52^{j}$ & $\begin{array}{l}31.60^{h} \\
37.31^{i}\end{array}$ \\
\hline $5 \mathrm{~s} 5 \mathrm{pz}^{3} \mathrm{P}_{2}^{\mathrm{o}}$ & 24647.121 & & $56.8 \pm 1.0^{e}$ & $75.66^{j}$ & $\begin{array}{l}33.41^{h} \\
42.74^{i}\end{array}$ \\
\hline $4 \mathrm{~d} 5 \mathrm{pz}^{1} \mathrm{D}_{2}^{\mathrm{o}}$ & 26147.252 & & $\begin{aligned} 5.9 & \pm 0.6^{d} \\
6.3 & \pm 0.2^{e} \\
6.82 & \pm 0.05^{f} \\
6.7 & \pm 0.5^{g}\end{aligned}$ & 5.94 & $\begin{array}{l}5.67^{h} \\
5.32^{i}\end{array}$ \\
\hline $4 d 5 \mathrm{pz}^{3} \mathrm{~F}_{2}^{\mathrm{o}}$ & 27227.027 & & $\begin{array}{l}6.8 \pm 0.8^{d} \\
6.3 \pm 0.3^{e}\end{array}$ & 5.67 & $\begin{array}{l}5.46^{h} \\
5.08^{i}\end{array}$ \\
\hline $4 \mathrm{~d} 5 \mathrm{pz}^{1} \mathrm{P}_{1}^{\mathrm{o}}$ & 27516.699 & & $\begin{array}{l}5.6 \pm 0.4^{d} \\
5.0 \pm 2.0^{e}\end{array}$ & 6.32 & $\begin{array}{l}5.58^{h} \\
4.93^{i}\end{array}$ \\
\hline $4 d 5 p z^{3} F_{3}^{o}$ & 27532.321 & & $\begin{array}{l}5.9 \pm 0.7^{d} \\
6.3 \pm 0.3^{e}\end{array}$ & 5.83 & $\begin{array}{l}5.42^{h} \\
5.21^{i}\end{array}$ \\
\hline $4 d 5 p z^{3} F_{4}^{o}$ & 28394.177 & & $\begin{array}{l}6.0 \pm 0.4^{d} \\
5.7 \pm 0.3^{e}\end{array}$ & 5.51 & $\begin{array}{l}5.17^{h} \\
4.91^{i}\end{array}$ \\
\hline $4 \mathrm{~d} 5 \mathrm{pz}^{3} \mathrm{D}_{1}^{\mathrm{o}}$ & 28595.285 & & $\begin{aligned} 4.5 & \pm 0.3^{e} \\
4.58 & \pm 0.05^{f}\end{aligned}$ & 4.08 & $\begin{array}{l}3.98^{h} \\
3.65^{i}\end{array}$ \\
\hline $4 \mathrm{~d} 5 \mathrm{pz}^{3} \mathrm{D}_{2}^{\mathrm{o}}$ & 28730.010 & & $\begin{aligned} 5.8 & \pm 0.8^{d} \\
4.3 & \pm 0.3^{e} \\
4.53 & \pm 0.09^{f} \\
6.4 & \pm 0.6^{g}\end{aligned}$ & 4.01 & $\begin{array}{l}3.80^{h} \\
3.52^{i}\end{array}$ \\
\hline $4 d 5 \mathrm{pz}^{3} \mathrm{D}_{3}^{\mathrm{o}}$ & 29213.958 & & $\begin{aligned} 5.2 & \pm 0.7^{d} \\
4.4 & \pm 0.3^{e} \\
4.43 & \pm 0.11^{f} \\
5.7 & \pm 0.8^{g}\end{aligned}$ & 3.93 & $\begin{array}{l}3.75^{h} \\
3.46^{i}\end{array}$ \\
\hline $4 \mathrm{~d} 5 \mathrm{py}^{3} \mathrm{P}_{0}^{\mathrm{o}}$ & 32048.788 & & $\begin{array}{c}3.4 \pm 0.2^{e} \\
2.87 \pm 0.04^{f} \\
2.8 \pm 0.2^{h}\end{array}$ & 2.55 & $\begin{array}{l}2.66^{h} \\
2.38^{i}\end{array}$ \\
\hline $4 d 5 p y^{3} P_{1}^{o}$ & 32124.054 & & $\begin{aligned} 4.2 & \pm 0.4^{d} \\
3.3 & \pm 0.2^{e} \\
2.87 & \pm 0.07^{f} \\
2.8 & \pm 0.2^{h}\end{aligned}$ & 2.55 & $\begin{array}{l}2.67^{h} \\
2.38^{i}\end{array}$ \\
\hline $4 d 5 p y^{3} P_{2}^{o}$ & 32283.420 & & $\begin{aligned} 3.8 & \pm 0.2^{d} \\
3.6 & \pm 0.2^{e} \\
3.08 & \pm 0.10^{f} \\
2.6 & \pm 0.2^{h}\end{aligned}$ & 2.53 & $\begin{array}{l}2.68^{h} \\
2.37^{i}\end{array}$ \\
\hline $4 \mathrm{~d} 5 \mathrm{pz}^{1} \mathrm{~F}_{3}^{\mathrm{o}}$ & 33336.727 & & $\begin{array}{c}6.9 \pm 0.7^{d} \\
5.49 \pm 0.09^{f} \\
4.7 \pm 0.3^{h}\end{array}$ & 5.08 & $\begin{array}{l}4.86^{h} \\
4.65^{i}\end{array}$ \\
\hline $5 \mathrm{~s} 5 \mathrm{py}^{1} \mathrm{P}_{1}^{\mathrm{o}}$ & 44568.540 & & $1.2 \pm 0.2^{h}$ & 1.05 & $\begin{array}{l}0.99^{h} \\
0.89^{i}\end{array}$ \\
\hline $4 d 6 \mathrm{se}^{3} \mathrm{D}_{1}$ & 54956.083 & $3.15 \pm 0.15$ & & 3.46 & $3.16^{i}$ \\
\hline $4 d 6 \mathrm{se}^{3} \mathrm{D}_{2}$ & 55032.349 & $3.17 \pm 0.15$ & & 3.61 & $3.28^{i}$ \\
\hline $4 d 6 \mathrm{se}^{3} \mathrm{D}_{3}$ & 55645.642 & $3.20 \pm 0.15$ & & 3.52 & $3.14^{i}$ \\
\hline $4 \mathrm{~d} 6 \mathrm{se}^{1} \mathrm{D}_{2}$ & 55725.522 & $3.14 \pm 0.15$ & & $4.38^{j}$ & $3.46^{i}$ \\
\hline $5 \mathrm{~s} 6 \mathrm{se}^{3} \mathrm{~S}_{1}$ & 58263.238 & $2.61 \pm 0.10$ & & 2.93 & $2.70^{i}$ \\
\hline $4 \mathrm{~d} 5 \mathrm{de}^{1} \mathrm{~F}_{3}$ & 58533.296 & $2.43 \pm 0.10$ & & 2.87 & $2.23^{i}$ \\
\hline $4 \mathrm{~d} 5 \mathrm{df}^{3} \mathrm{D}_{1}$ & 58720.382 & $2.60 \pm 0.15$ & & 2.88 & $2.40^{i}$ \\
\hline $5 \mathrm{p}^{2} \mathrm{e}^{3} \mathrm{P}_{0}$ & 58776.425 & $1.77 \pm 0.09$ & & 1.96 & $2.20^{i}$ \\
\hline $4 d 5 \mathrm{df}^{3} \mathrm{D}_{2}$ & 58947.625 & $2.53 \pm 0.10$ & & 2.95 & $2.56^{i}$ \\
\hline $5 \mathrm{p}^{2} \mathrm{e}^{3} \mathrm{P}_{1}$ & 59147.559 & $1.92 \pm 0.10$ & & 2.00 & $2.24^{i}$ \\
\hline $4 \mathrm{~d} 5 \mathrm{de}^{3} \mathrm{G}_{3}$ & 59179.589 & $2.53 \pm 0.15$ & & 2.72 & $2.13^{i}$ \\
\hline $4 \mathrm{~d} 5 \mathrm{df}^{3} \mathrm{D}_{3}$ & 59327.880 & $2.64 \pm 0.15$ & & 3.00 & $2.51^{i}$ \\
\hline $4 \mathrm{~d} 5 \mathrm{de}^{3} \mathrm{G}_{4}$ & 59472.643 & $2.45 \pm 0.15$ & & 2.75 & $2.14^{i}$ \\
\hline $5 \mathrm{p}^{2} \mathrm{e}^{3} \mathrm{P}_{2}$ & 59670.257 & $2.29 \pm 0.10$ & & 2.10 & $2.39^{i}$ \\
\hline $4 d 5 \mathrm{de}^{1} \mathrm{P}_{1}$ & 59716.843 & $2.64 \pm 0.10$ & & 3.03 & $2.40^{i}$ \\
\hline $4 \mathrm{~d} 5 \mathrm{de}^{3} \mathrm{G}_{5}$ & 59900.516 & $2.59 \pm 0.10$ & & 2.81 & $2.19^{i}$ \\
\hline $5 \mathrm{p}^{2} \mathrm{f}^{1} \mathrm{D}_{2}$ & 60535.922 & $4.36 \pm 0.20$ & & $5.55^{j}$ & $5.00^{i}$ \\
\hline $4 d 5 \mathrm{df}^{3} \mathrm{P}_{1}$ & 64263.741 & $1.30 \pm 0.07$ & & 1.60 & $0.94^{i}$ \\
\hline $4 \mathrm{~d} 5 \mathrm{df}^{3} \mathrm{P}_{2}$ & 64597.237 & $1.23 \pm 0.05$ & & 1.55 & $0.93^{i}$ \\
\hline
\end{tabular}


Table 2 - continued

\begin{tabular}{|c|c|c|c|c|c|}
\hline \multirow[t]{2}{*}{ Level $^{a}$} & \multirow{2}{*}{$\begin{array}{c}\text { Energy }^{a} \\
\left(\mathrm{~cm}^{-1}\right)\end{array}$} & \multicolumn{2}{|c|}{ Experimental lifetime (ns) } & \multicolumn{2}{|c|}{ Theoretical lifetime (ns) } \\
\hline & & This work ${ }^{b}$ & Other works & This work $^{c}$ & Other works \\
\hline $4 d 7 s^{3} D_{3}$ & 74374.907 & $4.11 \pm 0.30$ & & $6.20^{j}$ & $5.99^{i}$ \\
\hline $4 d 7 s^{1} D_{2}$ & 74582.562 & $4.40 \pm 0.30$ & & $6.32^{j}$ & $6.10^{i}$ \\
\hline $4 d 6 d^{3} D_{3}$ & 76178.282 & $3.76 \pm 0.20$ & & $8.56^{j}$ & $7.25^{i}$ \\
\hline
\end{tabular}

Notes. ${ }^{\text {a} N i l s s o n ~ e t ~ a l . ~(1991) . ~}$

${ }^{\mathrm{b}}$ TR-LIF measurements (see the text).

${ }^{\mathrm{c}} \mathrm{HFR}+\mathrm{CPOL}$ calculations (see the text).

${ }^{\mathrm{d}}$ Gorshklov \& Komarovskii (1986), retarded coincidence in intersecting atomic and electron beams

${ }^{\text {e}}$ Hannaford et al. (1982), laser-induced fluorescence on sputtered metal vapour.

${ }^{\mathrm{f}}$ Wännström et al. (1988), beam-laser technique.

${ }^{\mathrm{g}}$ Andersen et al. (1978), beam-foil and sputtering excitation techniques.

${ }^{\mathrm{h}}$ Biémont et al. (2011), laser-induced fluorescence on laser produced plasma.

${ }^{\mathrm{i}}$ Kurucz (2011), semi-empirical calculations.

${ }^{j}$ Affected by strong cancellation effects, see discussion in text.

$4 d 5 p{ }^{3} D_{3}$ level is sufficiently close to the decay of the $4 d 7 s{ }^{3} D_{3}$ level, which we measured in the second spectral order at $474 \mathrm{~nm}$, to give a noticeable contribution to the decay curve, as seen in Fig. 1. However, this can be accurately corrected for by recording a separate decay curve with the second-step laser blocked, which is then subtracted from the first measurement before the lifetime analysis. All levels were checked for this effect. Several other cases were encountered and corrected for in a similar way, as noted in Table 1.

A more serious problem is caused by so-called cascades. One example encountered in this work is in the decay of the $4 d 5 d{ }^{3} \mathrm{D}_{1}$ level at $58720 \mathrm{~cm}^{-1}$. Here, we measured in two channels, 306.9 and $374.8 \mathrm{~nm}$, but had to omit a third possibility at $320.4 \mathrm{~nm}$ since this line is blended by a cascade transition at $320.3 \mathrm{~nm}$ arising from $5 \mathrm{~d} 5 \mathrm{p}^{3} \mathrm{P}_{0}$ populated from the $4 \mathrm{~d} 5 \mathrm{~d}^{3} \mathrm{D}_{1}$ level by the $374.8 \mathrm{~nm}$ transition. Since such problems cannot be corrected, spectroscopic investigations must be made to avoid using any perturbed channels. In this respect the availability of the comprehensive term analysis of Y II by Nilsson, Johansson \& Kurucz (1991) is invaluable, since it allows us to identify which decay channels might be affected.

\section{HFR +CPOL CALCULATIONS}

As our previous calculations in Y II (Biémont et al. 2011) were restricted to correlation up to $n=6$, the present HFR+CPOL calculations have been extended to $n=10$ to model the highly excited energy levels up to $n=7$ measured in this study.

The pseudo-relativistic Hartree-Fock (HFR) method (Cowan 1981) incorporating a core-polarization correction (CPOL) to the Hartree-Fock potential and to the dipole operator (Quinet et al. 1999, 2002) has been used. The configurations considered in the configuration interaction (CI) expansions were the following: $5 \mathrm{~s}^{2}+5 \mathrm{sns}(n=6-10)+5 \mathrm{snd}(n=4-10)+5 \mathrm{sng}$ $(n=5-10)+4 \mathrm{~d}^{2}+4 \mathrm{dns}(n=6-10)+4 \mathrm{dnd}(n=5-10)+$ $4 \mathrm{dng}(n=5-10)+5 \mathrm{~d}^{2}+5 \mathrm{~d} 6 \mathrm{~s}+5 \mathrm{~d} 6 \mathrm{~d}+5 \mathrm{p}^{2}+5 \mathrm{png}(n=$ $4-6)+6 \mathrm{~s}^{2}+6 \mathrm{p}^{2}+6 \mathrm{pnf}(n=4-6)$ for the even parity; $5 \mathrm{snp}$ $(n=5-10)+5 \operatorname{snf}(n=4-10)+5 \operatorname{snh}(n=6-10)+4 \operatorname{dnp}(n$ $=5-10)+4 \operatorname{dnf}(n=4-10)+4 \operatorname{dnh}(n=6-10)+5$ pnd $(n$ $=5-6)+6$ pnd $(n=5-6)$ for the odd parity. The ionic core considered for the core-polarization effects was a krypton-like yttrium [Ar] $3 \mathrm{~d}^{10} 4 \mathrm{~s}^{2} 4 \mathrm{p}^{6}$ core with a static dipole polarizability of $\alpha_{c}=4.05 a_{0}^{3}$ (Johnsson, Kolb \& Huang 1983) and a cut-off radius taken as the HFR mean radius of the outermost core orbital, i.e. $r_{c}=\langle 4 p|r| 4 p\rangle_{\mathrm{HFR}}=1.453 a_{0}$.
In a least-squares fitting procedure, some radial parameters have been adjusted to minimize the differences between the Hamiltonian eigenvalues and the experimental energy levels of Nilsson et al. (1991). The levels belong to the configurations $5 \mathrm{~s}^{2}, 5 \mathrm{sns} n=6-8$, $5 \operatorname{snp} n=5-6,5 \operatorname{snd} n=4-6,5 \operatorname{snf} n=4-5,4 \mathrm{~d}^{2}, 5 \mathrm{dns} n=6-9$, $5 \mathrm{dnp} n=5-7,5 \mathrm{dnd} n=5-8,5 \mathrm{dnf} n=4-7,5 \mathrm{~d} 5 \mathrm{~g}$ and $5 \mathrm{p}^{2}$. The configuration average energies, $E_{\mathrm{av}}$, the direct and exchange Slater integrals $F^{k}$ and $G^{k}$, the effective interaction parameters $(\alpha$, $\beta$ and $T$ ) and the spin-orbit integrals $\zeta$ of these configurations have been fitted. Their fitted and ab initio values are reported in Table 3. All the other Slater integrals have been scaled down by a factor of 0.85 .

In total, 119 even-parity and 115 odd-parity experimental energy levels published in Nilsson et al. (1991) have been included in the fitting procedure and the average deviations have been minimized to $158 \mathrm{~cm}^{-1}$ for the even-parity levels and to $118 \mathrm{~cm}^{-1}$ for the odd-parity levels.

\section{RESULTS AND DISCUSSION}

Our lifetimes are given in Table 2 and compared to available experimental and theoretical values.

For the odd-parity levels, our theoretical values are, in most of the cases, slightly larger than our previous calculations (Biémont et al. 2011), i.e. they are $\sim 5$ to $\sim 15$ per cent larger with the exception of the triplets $5 \mathrm{~s} 5 \mathrm{p} \mathrm{z}^{3} \mathrm{P}^{o}$ and $4 \mathrm{~d} 5 \mathrm{p} \mathrm{y}^{3} \mathrm{P}^{o}$, and generally in better agreement with measurements. Some of the theoretical lifetimes are affected by strong cancellation effects (with cancellation factors as defined by Cowan 1981 less than 0.1) on decay channels that contribute significantly (more than 10 per cent) to the radiative lifetime. They are marked with an asterisk in Table 2 and are model sensitive. For instance, the three theoretical values are noticeably different for the level $5 \mathrm{~s} 5 \mathrm{p} \mathrm{z}^{3} \mathrm{P}_{2}^{o}$ and the cancellation effects tend to lengthen the calculated lifetimes.

For the even-parity levels, our calculated values are on average slightly longer than our experimental ones by about 10 per cent. This means that the core-polarization effects are overestimated for the even-parity levels in our model. On the other hand, the lifetimes calculated by Kurucz (2011), who used Cowan's codes (Cowan 1981), are on average 5 per cent shorter than our measurements.

As for the odd levels, some lifetimes are significantly longer than our measurements by up to a factor two, notably for the level $4 \mathrm{~d}^{3} \mathrm{~d}^{3} \mathrm{D}_{3}$. In our calculations, this is due to strong cancellation effects. Most likely this is also the case for the Kurucz data, although 
Table 3. Radial parameters adopted in our HFR+CPOL model of the Y II atomic structure. All Slater and spin-orbit parameters not listed here have been respectively scaled down by 0.85 and fixed to their ab initio values.

\begin{tabular}{|c|c|c|c|c|c|}
\hline Configuration & Parameter & $\begin{array}{l}\text { Ab initio } \\
\left(\mathrm{cm}^{-1}\right)\end{array}$ & $\begin{array}{l}\text { Fitted } \\
\left(\mathrm{cm}^{-1}\right)\end{array}$ & Ratio & Note $^{a}$ \\
\hline $5 s^{2}$ & $E_{\mathrm{av}}$ & $\begin{array}{l}\text { Even pa } \\
4830\end{array}$ & 4944 & & \\
\hline $5 \mathrm{~s} 6 \mathrm{~s}$ & $\begin{array}{c}E_{\mathrm{av}} \\
G^{0}(5 \mathrm{~s} 6 \mathrm{~s})\end{array}$ & $\begin{array}{c}59972 \\
2059\end{array}$ & $\begin{array}{c}60082 \\
1740\end{array}$ & 0.845 & \\
\hline $5 \mathrm{~s} 7 \mathrm{~s}$ & $\begin{array}{c}E_{\mathrm{av}} \\
G^{0}(5 \mathrm{~s} 7 \mathrm{~s})\end{array}$ & $\begin{array}{c}80350 \\
666\end{array}$ & $\begin{array}{c}80089 \\
579\end{array}$ & 0.850 & $\mathrm{~F}$ \\
\hline $5 \mathrm{~s} 8 \mathrm{~s}$ & $\begin{array}{c}E_{\mathrm{av}} \\
G^{0}(5 \mathrm{~s} 8 \mathrm{~s})\end{array}$ & $\begin{array}{c}89519 \\
312\end{array}$ & $\begin{array}{c}89339 \\
271\end{array}$ & 0.850 & $\mathrm{~F}$ \\
\hline $5 \mathrm{~s} 4 \mathrm{~d}$ & $\begin{array}{c}E_{\mathrm{av}} \\
\zeta_{4 \mathrm{~d}} \\
G^{2}(5 \mathrm{~s} 4 \mathrm{~d})\end{array}$ & $\begin{array}{c}2620 \\
287 \\
16294\end{array}$ & $\begin{array}{c}2905 \\
229 \\
15118\end{array}$ & $\begin{array}{l}0.798 \\
0.928\end{array}$ & $\mathrm{R} 1$ \\
\hline $5 \mathrm{~s} 5 \mathrm{~d}$ & $\begin{array}{c}E_{\mathrm{av}} \\
\zeta 5 \mathrm{~d} \\
G^{2}(5 \mathrm{~s} 5 \mathrm{~d})\end{array}$ & $\begin{array}{c}65311 \\
42 \\
3580\end{array}$ & $\begin{array}{c}65942 \\
42 \\
3321\end{array}$ & $\begin{array}{l}1.000 \\
0.928\end{array}$ & $\begin{array}{c}\mathrm{F} \\
\mathrm{R} 1\end{array}$ \\
\hline $5 \mathrm{~s} 6 \mathrm{~d}$ & $\begin{array}{c}E_{\mathrm{av}} \\
\zeta_{6 \mathrm{~d}} \\
G^{2}(5 \mathrm{~s} 6 \mathrm{~d})\end{array}$ & $\begin{array}{c}82850 \\
17 \\
1298\end{array}$ & $\begin{array}{c}82936 \\
17 \\
1204\end{array}$ & $\begin{array}{l}1.000 \\
0.928\end{array}$ & $\begin{array}{c}\mathrm{F} \\
\mathrm{R} 1\end{array}$ \\
\hline $4 d^{2}$ & $\begin{array}{c}E_{\mathrm{av}} \\
F^{2}(4 \mathrm{~d} 4 \mathrm{~d}) \\
F^{4}(4 \mathrm{~d} 4 \mathrm{~d}) \\
\alpha \\
\beta \\
T \\
\zeta_{4 \mathrm{~d}}\end{array}$ & $\begin{array}{c}12456 \\
38633 \\
24710 \\
0 \\
0 \\
0 \\
244\end{array}$ & $\begin{array}{c}12295 \\
31330 \\
20257 \\
43 \\
-879 \\
3 \\
154\end{array}$ & $\begin{array}{l}0.811 \\
0.820\end{array}$ & \\
\hline $4 \mathrm{~d} 6 \mathrm{~s}$ & $\begin{array}{c}E_{\mathrm{av}} \\
\zeta_{4 \mathrm{~d}} \\
G^{2}(4 \mathrm{~d} 6 \mathrm{~s})\end{array}$ & $\begin{array}{c}55205 \\
313 \\
2527\end{array}$ & $\begin{array}{c}56380 \\
215 \\
2344\end{array}$ & $\begin{array}{l}0.687 \\
0.928\end{array}$ & $\begin{array}{l}\mathrm{R} 2 \\
\mathrm{R} 1\end{array}$ \\
\hline $4 \mathrm{~d} 7 \mathrm{~s}$ & $\begin{array}{c}E_{\mathrm{av}} \\
\zeta_{4 \mathrm{~d}} \\
G^{2}(4 \mathrm{~d} 7 \mathrm{~s})\end{array}$ & $\begin{array}{c}73557 \\
317 \\
933\end{array}$ & $\begin{array}{c}74073 \\
218 \\
865\end{array}$ & $\begin{array}{l}0.687 \\
0.928\end{array}$ & $\begin{array}{l}\mathrm{R} 2 \\
\mathrm{R} 1\end{array}$ \\
\hline $4 \mathrm{~d} 8 \mathrm{~s}$ & $\begin{array}{c}E_{\mathrm{av}} \\
\zeta_{4 \mathrm{~d}} \\
G^{2}(4 \mathrm{~d} 8 \mathrm{~s})\end{array}$ & $\begin{array}{c}82166 \\
318 \\
460\end{array}$ & $\begin{array}{c}82674 \\
218 \\
426\end{array}$ & $\begin{array}{l}0.687 \\
0.928\end{array}$ & $\begin{array}{l}\mathrm{R} 2 \\
\mathrm{R} 1\end{array}$ \\
\hline $4 \mathrm{~d} 9 \mathrm{~s}$ & $\begin{array}{c}E_{\mathrm{av}} \\
\zeta_{4 \mathrm{~d}} \\
G^{2}(4 \mathrm{~d} 9 \mathrm{~s})\end{array}$ & $\begin{array}{c}86934 \\
319 \\
262\end{array}$ & $\begin{array}{c}87585 \\
219 \\
244\end{array}$ & $\begin{array}{l}0.687 \\
0.928\end{array}$ & $\begin{array}{l}\mathrm{R} 2 \\
\mathrm{R} 1\end{array}$ \\
\hline $4 d 5 d$ & $\begin{array}{c}E_{\mathrm{av}} \\
\zeta_{4 \mathrm{~d}} \\
\zeta_{5 \mathrm{~d}} \\
F^{2}(4 \mathrm{~d} 5 \mathrm{~d}) \\
F^{4}(4 \mathrm{~d} 5 \mathrm{~d}) \\
G^{0}(4 \mathrm{~d} 5 \mathrm{~d}) \\
G^{2}(4 \mathrm{~d} 5 \mathrm{~d}) \\
G^{4}(4 \mathrm{~d} 5 \mathrm{~d})\end{array}$ & $\begin{array}{c}59997 \\
313 \\
37 \\
7388 \\
3452 \\
4181 \\
3330 \\
2451\end{array}$ & $\begin{array}{c}61257 \\
302 \\
37 \\
4988 \\
2331 \\
1958 \\
1559 \\
1148\end{array}$ & $\begin{array}{l}0.965 \\
1.000 \\
0.675 \\
0.675 \\
0.468 \\
0.468 \\
0.468\end{array}$ & $\begin{array}{c}\text { R3 } \\
\text { F } \\
\text { R4 } \\
\text { R4 } \\
\text { R5 } \\
\text { R5 } \\
\text { R5 }\end{array}$ \\
\hline $4 \mathrm{~d} 6 \mathrm{~d}$ & $\begin{array}{c}E_{\mathrm{av}} \\
\zeta_{4 \mathrm{~d}} \\
\zeta_{6 \mathrm{~d}} \\
F^{2}(4 \mathrm{~d} 6 \mathrm{~d}) \\
F^{4}(4 \mathrm{~d} 6 \mathrm{~d}) \\
G^{0}(4 \mathrm{~d} 6 \mathrm{~d}) \\
G^{2}(4 \mathrm{~d} 6 \mathrm{~d}) \\
G^{4}(4 \mathrm{~d} 6 \mathrm{~d})\end{array}$ & $\begin{array}{c}75859 \\
317 \\
15 \\
2847 \\
1323 \\
1434 \\
1241 \\
938\end{array}$ & $\begin{array}{c}76512 \\
305 \\
15 \\
1923 \\
892 \\
672 \\
581 \\
439\end{array}$ & $\begin{array}{l}0.965 \\
1.000 \\
0.675 \\
0.675 \\
0.468 \\
0.468 \\
0.468\end{array}$ & $\begin{array}{c}\text { R3 } \\
\text { F } \\
\text { R4 } \\
\text { R4 } \\
\text { R5 } \\
\text { R5 } \\
\text { R5 }\end{array}$ \\
\hline $4 d 7 d$ & $\begin{array}{l}E_{\mathrm{av}} \\
\zeta_{4 \mathrm{~d}} \\
\zeta_{7 \mathrm{~d}}\end{array}$ & $\begin{array}{c}83423 \\
318 \\
8\end{array}$ & $\begin{array}{c}84070 \\
306 \\
8\end{array}$ & $\begin{array}{l}0.965 \\
1.000\end{array}$ & $\begin{array}{l}\mathrm{R} 3 \\
\mathrm{~F}\end{array}$ \\
\hline
\end{tabular}


Table 3 - continued

\begin{tabular}{|c|c|c|c|c|c|}
\hline Configuration & Parameter & $\begin{array}{l}\text { Ab initio } \\
\left(\mathrm{cm}^{-1}\right)\end{array}$ & $\begin{array}{l}\text { Fitted } \\
\left(\mathrm{cm}^{-1}\right)\end{array}$ & Ratio & Note $^{a}$ \\
\hline & $\begin{array}{l}F^{2}(4 \mathrm{~d} 7 \mathrm{~d}) \\
F^{4}(4 \mathrm{~d} 7 \mathrm{~d}) \\
G^{0}(4 \mathrm{~d} 7 \mathrm{~d}) \\
G^{2}(4 \mathrm{~d} 7 \mathrm{~d}) \\
G^{4}(4 \mathrm{~d} 7 \mathrm{~d})\end{array}$ & $\begin{array}{c}1440 \\
676 \\
696 \\
624 \\
477\end{array}$ & $\begin{array}{l}972 \\
456 \\
325 \\
292 \\
224\end{array}$ & $\begin{array}{l}0.675 \\
0.675 \\
0.468 \\
0.468 \\
0.468\end{array}$ & $\begin{array}{l}\text { R4 } \\
\text { R4 } \\
\text { R5 } \\
\text { R5 } \\
\text { R5 }\end{array}$ \\
\hline $4 \mathrm{~d} 8 \mathrm{~d}$ & $\begin{array}{c}E_{\mathrm{av}} \\
\zeta_{4 \mathrm{~d}} \\
\zeta_{8 \mathrm{~d}} \\
F^{2}(4 \mathrm{~d} 8 \mathrm{~d}) \\
F^{4}(4 \mathrm{~d} 8 \mathrm{~d}) \\
G^{0}(4 \mathrm{~d} 8 \mathrm{~d}) \\
G^{2}(4 \mathrm{~d} 8 \mathrm{~d}) \\
G^{4}(4 \mathrm{~d} 8 \mathrm{~d})\end{array}$ & $\begin{array}{c}87692 \\
319 \\
5 \\
836 \\
396 \\
396 \\
362 \\
278\end{array}$ & $\begin{array}{c}88413 \\
307 \\
5 \\
565 \\
268 \\
185 \\
170 \\
130\end{array}$ & $\begin{array}{l}0.965 \\
1.000 \\
0.675 \\
0.675 \\
0.468 \\
0.468 \\
0.468\end{array}$ & $\begin{array}{c}\text { R3 } \\
\text { F } \\
\text { R4 } \\
\text { R4 } \\
\text { R5 } \\
\text { R5 } \\
\text { R5 }\end{array}$ \\
\hline $4 \mathrm{~d} 5 \mathrm{~g}$ & $\begin{array}{c}E_{\mathrm{av}} \\
\zeta_{4 \mathrm{~d}} \\
\zeta_{5 \mathrm{~g}} \\
F^{2}(4 \mathrm{~d} 5 \mathrm{~g}) \\
F^{4}(4 \mathrm{~d} 5 \mathrm{~g}) \\
G^{2}(4 \mathrm{~d} 5 \mathrm{~g}) \\
G^{4}(4 \mathrm{~d} 5 \mathrm{~g}) \\
G^{6}(4 \mathrm{~d} 5 \mathrm{~g})\end{array}$ & $\begin{array}{c}80522 \\
319 \\
0 \\
906 \\
133 \\
32 \\
22 \\
16\end{array}$ & $\begin{array}{c}81390 \\
319 \\
0 \\
788 \\
116 \\
28 \\
19 \\
14\end{array}$ & $\begin{array}{l}1.000 \\
1.000 \\
0.850 \\
0.850 \\
0.850 \\
0.850 \\
0.850\end{array}$ & $\begin{array}{l}F \\
F \\
F \\
F \\
F \\
F \\
F\end{array}$ \\
\hline $5 p^{2}$ & $\begin{array}{c}E_{\mathrm{av}} \\
F^{2}(5 \mathrm{p} 5 \mathrm{p}) \\
\alpha \\
\zeta_{5 \mathrm{p}}\end{array}$ & $\begin{array}{c}61417 \\
25147 \\
0 \\
658\end{array}$ & $\begin{array}{c}62631 \\
16038 \\
0 \\
634\end{array}$ & $\begin{array}{l}0.638 \\
0.964\end{array}$ & $\mathrm{~F}$ \\
\hline \multicolumn{6}{|c|}{ Odd parity } \\
\hline $5 s 5 p$ & $\begin{array}{c}E_{\mathrm{av}} \\
\zeta_{5 \mathrm{p}} \\
G^{1}(5 \mathrm{~s} 5 \mathrm{p})\end{array}$ & $\begin{array}{c}27694 \\
654 \\
31781\end{array}$ & $\begin{array}{c}29865 \\
960 \\
23177\end{array}$ & $\begin{array}{l}1.468 \\
0.729\end{array}$ & $\begin{array}{l}\text { R6 } \\
\text { R7 }\end{array}$ \\
\hline $5 s 6 p$ & $\begin{array}{c}E_{\mathrm{av}} \\
\zeta_{6 \mathrm{p}} \\
G^{1}(5 \mathrm{~s} 6 \mathrm{p})\end{array}$ & $\begin{array}{c}69782 \\
198 \\
3948\end{array}$ & $\begin{array}{c}69960 \\
291 \\
2879\end{array}$ & $\begin{array}{l}1.468 \\
0.729\end{array}$ & $\begin{array}{l}\text { R6 } \\
\text { R7 }\end{array}$ \\
\hline $5 \mathrm{~s} 4 \mathrm{f}$ & $\begin{array}{c}E_{\mathrm{av}} \\
\zeta_{4 \mathrm{f}} \\
G^{3}(5 \mathrm{~s} 4 \mathrm{f})\end{array}$ & $\begin{array}{c}76144 \\
0 \\
4283\end{array}$ & $\begin{array}{c}77227 \\
0 \\
3222\end{array}$ & $\begin{array}{l}1.000 \\
0.752\end{array}$ & $\begin{array}{c}\mathrm{F} \\
\mathrm{R} 8\end{array}$ \\
\hline $5 \mathrm{~s} 5 \mathrm{f}$ & $\begin{array}{c}E_{\mathrm{av}} \\
\zeta_{5 \mathrm{f}} \\
G^{3}(5 \mathrm{~s} 5 \mathrm{f})\end{array}$ & $\begin{array}{c}87425 \\
0 \\
2138\end{array}$ & $\begin{array}{c}87617 \\
0 \\
1609\end{array}$ & $\begin{array}{l}1.000 \\
0.752\end{array}$ & $\begin{array}{c}\mathrm{F} \\
\mathrm{R} 8\end{array}$ \\
\hline $4 d 5 p$ & $\begin{array}{c}E_{\mathrm{av}} \\
\zeta_{4 \mathrm{~d}} \\
\zeta_{5 \mathrm{p}} \\
F^{2}(4 \mathrm{~d} 5 \mathrm{p}) \\
G^{1}(4 \mathrm{~d} 5 \mathrm{p}) \\
G^{3}(4 \mathrm{~d} 5 \mathrm{p})\end{array}$ & $\begin{array}{c}28527 \\
299 \\
523 \\
16960 \\
9651 \\
7271\end{array}$ & $\begin{array}{c}29831 \\
259 \\
637 \\
13743 \\
8517 \\
6418\end{array}$ & $\begin{array}{l}0.866 \\
1.218 \\
0.810 \\
0.883 \\
0.883\end{array}$ & $\begin{array}{l}\text { R9 } \\
\text { R10 } \\
\text { R11 } \\
\text { R12 } \\
\text { R12 }\end{array}$ \\
\hline $4 d 6 p$ & $\begin{array}{c}E_{\mathrm{av}} \\
\zeta_{4 \mathrm{~d}} \\
\zeta_{6 \mathrm{p}} \\
F^{2}(4 \mathrm{~d} 6 \mathrm{p}) \\
G^{1}(4 \mathrm{~d} 6 \mathrm{p}) \\
G^{3}(4 \mathrm{~d} 6 \mathrm{p})\end{array}$ & $\begin{array}{c}63890 \\
314 \\
180 \\
4914 \\
1939 \\
1674\end{array}$ & $\begin{array}{c}64656 \\
273 \\
219 \\
3982 \\
1711 \\
1478\end{array}$ & $\begin{array}{l}0.866 \\
1.218 \\
0.810 \\
0.883 \\
0.883\end{array}$ & $\begin{array}{l}\text { R9 } \\
\text { R10 } \\
\text { R11 } \\
\text { R12 } \\
\text { R12 }\end{array}$ \\
\hline $4 d 7 p$ & $\begin{array}{c}E_{\mathrm{av}} \\
\zeta_{4 \mathrm{~d}} \\
\zeta_{7 \mathrm{p}} \\
F^{2}(4 \mathrm{~d} 7 \mathrm{p}) \\
G^{1}(4 \mathrm{~d} 7 \mathrm{p}) \\
G^{3}(4 \mathrm{~d} 7 \mathrm{p})\end{array}$ & $\begin{array}{c}77473 \\
317 \\
84 \\
2101 \\
784 \\
702\end{array}$ & $\begin{array}{c}78090 \\
275 \\
102 \\
1702 \\
692 \\
619\end{array}$ & $\begin{array}{l}0.866 \\
1.218 \\
0.810 \\
0.883 \\
0.883\end{array}$ & $\begin{array}{l}\text { R9 } \\
\text { R10 } \\
\text { R11 } \\
\text { R12 } \\
\text { R12 }\end{array}$ \\
\hline
\end{tabular}


Table 3 - continued

\begin{tabular}{|c|c|c|c|c|c|}
\hline Configuration & Parameter & $\begin{array}{c}\text { Ab initio } \\
\left(\mathrm{cm}^{-1}\right)\end{array}$ & $\begin{array}{l}\text { Fitted } \\
\left(\mathrm{cm}^{-1}\right)\end{array}$ & Ratio & Note $^{a}$ \\
\hline \multirow[t]{8}{*}{$4 d 4 f$} & $E_{\mathrm{av}}$ & 69478 & 70790 & & \\
\hline & $\zeta_{4 \mathrm{~d}}$ & 317 & 292 & 0.921 & $\mathrm{R} 13$ \\
\hline & $\zeta_{4 \mathrm{f}}$ & 0 & 0 & 1.000 & $\mathrm{~F}$ \\
\hline & $F^{2}(4 \mathrm{~d} 4 \mathrm{f})$ & 4349 & 3265 & 0.751 & $\mathrm{R} 14$ \\
\hline & $F^{4}(4 \mathrm{~d} 4 \mathrm{f})$ & 1534 & 1151 & 0.751 & $\mathrm{R} 14$ \\
\hline & $G^{1}(4 \mathrm{~d} 4 \mathrm{f})$ & 1743 & 1253 & 0.719 & $\mathrm{R} 15$ \\
\hline & $G^{3}(4 d 4 f)$ & 1014 & 729 & 0.719 & $\mathrm{R} 15$ \\
\hline & $G^{5}(4 d 4 f)$ & 699 & 502 & 0.719 & $\mathrm{R} 15$ \\
\hline \multirow[t]{8}{*}{$4 \mathrm{~d} 5 \mathrm{f}$} & $E_{\mathrm{av}}$ & 80041 & 80958 & & \\
\hline & $\zeta_{4 \mathrm{~d}}$ & 318 & 293 & 0.921 & $\mathrm{R} 13$ \\
\hline & $\zeta_{5 \mathrm{f}}$ & 0 & 0 & 1.000 & $\mathrm{~F}$ \\
\hline & $F^{2}(4 \mathrm{~d} 5 \mathrm{f})$ & 2069 & 1553 & 0.751 & $\mathrm{R} 14$ \\
\hline & $F^{4}(4 \mathrm{~d} 5 \mathrm{f})$ & 820 & 615 & 0.751 & R14 \\
\hline & $G^{1}(4 \mathrm{~d} 5 \mathrm{f})$ & 1087 & 781 & 0.719 & $\mathrm{R} 15$ \\
\hline & $G^{3}(4 \mathrm{~d} 5 \mathrm{f})$ & 639 & 459 & 0.719 & $\mathrm{R} 15$ \\
\hline & $G^{5}(4 \mathrm{~d} 5 \mathrm{f})$ & 443 & 318 & 0.719 & $\mathrm{R} 15$ \\
\hline \multirow[t]{8}{*}{$4 d 6 f$} & $E_{\mathrm{av}}$ & 85675 & 86502 & & \\
\hline & $\zeta_{4 \mathrm{~d}}$ & 318 & 294 & 0.921 & $\mathrm{R} 13$ \\
\hline & $\zeta_{6 f}$ & 0 & 0 & 1.000 & $\mathrm{~F}$ \\
\hline & $F^{2}(4 \mathrm{~d} 6 \mathrm{f})$ & 1165 & 875 & 0.751 & $\mathrm{R} 14$ \\
\hline & $F^{4}(4 \mathrm{~d} 6 \mathrm{f})$ & 488 & 366 & 0.751 & R14 \\
\hline & $G^{1}(4 \mathrm{~d} 6 \mathrm{f})$ & 680 & 488 & 0.719 & $\mathrm{R} 15$ \\
\hline & $G^{3}(4 \mathrm{~d} 6 \mathrm{f})$ & 403 & 289 & 0.719 & $\mathrm{R} 15$ \\
\hline & $G^{5}(4 \mathrm{~d} 6 \mathrm{f})$ & 278 & 200 & 0.719 & $\mathrm{R} 15$ \\
\hline \multirow[t]{8}{*}{$4 d 7 f$} & $E_{\mathrm{av}}$ & 89048 & 89844 & & \\
\hline & $\zeta_{4 \mathrm{~d}}$ & 319 & 294 & 0.921 & $\mathrm{R} 13$ \\
\hline & $\zeta_{7 f}$ & 0 & 0 & 1.000 & $\mathrm{~F}$ \\
\hline & $F^{2}(4 \mathrm{~d} 7 \mathrm{f})$ & 722 & 542 & 0.751 & R14 \\
\hline & $F^{4}(4 \mathrm{~d} 7 \mathrm{f})$ & 311 & 233 & 0.751 & R14 \\
\hline & $G^{1}(4 \mathrm{~d} 7 \mathrm{f})$ & 445 & 320 & 0.719 & $\mathrm{R} 15$ \\
\hline & $G^{3}(4 \mathrm{~d} 7 \mathrm{f})$ & 265 & 190 & 0.719 & $\mathrm{R} 15$ \\
\hline & $G^{5}(4 \mathrm{~d} 7 \mathrm{f})$ & 183 & 132 & 0.719 & $\mathrm{R} 15$ \\
\hline
\end{tabular}

${ }^{a} \mathrm{~F}$ : fixed parameter value; $\mathrm{R} n$ : fixed ratio between these parameters.

the cancellation factors are not available in Kurucz's data base (Kurucz 2011).

Table 4 is a sample of a bigger table listing the strongest 357 E1 decay channels (having an $A$-value greater than $10^{4} \mathrm{~s}^{-1}$ ) depopulating the levels for which the lifetime has ever been mea-

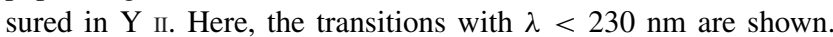
The whole table is available in electronic format at the Centre de Données astronomiques de Strasbourg (CDS 2017) and in the online version of the paper as supplementary material. Along with the HFR+CPOL oscillator strengths $(\log g f)$ and transition probabilities $(g A)$, the corresponding corrected radiative parameters $\left(\log g f_{\mathrm{c}}\right.$ and $\left.g A_{\mathrm{c}}\right)$ are given for each transition with the experimental lifetime of the upper level $\left(\tau_{\mathrm{c}}\right)$ used to rescale these parameters. We recommend the astronomical community to use these rescaled values as they should correct the overestimation of the core-polarization effects by our model for the highlyexcited even-parity levels involved in the transition outlined in the previous paragraph.

In Figs 2-4, the present HFR+CPOL oscillator strengths are compared with our previous values (Biémont et al. 2011), those of Kurucz (Kurucz 2011) and the experimental values of Hannaford et al. (1982), respectively. Although the latter concerns exclusively the decay transitions of low-lying odd-parity levels (Hannaford et al. 1982), they nonetheless provide a good test of the present HFR+CPOL model.

Fig. 2 shows a good agreement between our two calculations with no systematic effects, as the core-polarization has been taken into account in both models. However, some discrepancies are seen for the weak transitions due to cancellations such as the transition $4 \mathrm{~d}^{2} \mathrm{a}^{2} \mathrm{P}_{0}-4 \mathrm{~d} 5 \mathrm{pz}^{1} \mathrm{P}_{1}^{\mathrm{o}}$ at $733.295 \mathrm{~nm}$ with $\log g f=-3.08$ and a cancellation factor of $\mathrm{CF}=0.07$ in this work, compared to $\log g f=-1.98$ obtained with our previous model. In this particular case, it is advisable to use our older published value (Biémont et al. 2011), i.e. -1.98 , that belongs to a smaller set of calculated strong (log $g f>-2)$ decay transitions being not affected by cancellation. Besides, Kurucz (2011) gives a value of -2.87 for that line. We suspect that this oscillator strength is also affected by cancellation as it is calculated $\sim 1$ dex weaker than the value of Biémont et al. (2011), similarly to the present calculation. Unfortunately, CF values are not reported in Kurucz (2011).

Fig. 3 shows that the oscillator strengths computed by Kurucz (2011) are systematically larger than ours by, on average, $0.07 \mathrm{dex}$ for lines with $\log g f>0$. Furthermore, a significant number $(92$ transitions out of 357) of the lines with $\log g f<0$ are affected by strong cancellation effects $(\mathrm{CF}<0.1)$ showing discrepancies of one dex or more. Using our $\log g f$-values with $\mathrm{CF}<0.1$ is not 
Table 4. Transition probabilities ( $g A$ ) and oscillator strengths ( $\log g f$ ) for the strongest (with an $A$-value greater than $10^{4} \mathrm{~s}^{-1}$ ) decay channels depopulating the levels for which the lifetime has been measured in Y II. This is a sample for the transitions with $\lambda<230 \mathrm{~nm}$. The complete table is available in electronic format at the CDS (2017) and in the online version of the paper as supplementary material.

\begin{tabular}{|c|c|c|c|c|c|c|c|c|c|c|c|c|c|}
\hline $\begin{array}{l}\lambda^{a} \\
(\mathrm{~nm})\end{array}$ & $\begin{array}{c}E_{\mathrm{low}}^{b} \\
\left(\mathrm{~cm}^{-1}\right)\end{array}$ & $P_{\text {low }}^{c}$ & $J_{\text {low }}$ & $\begin{array}{c}E_{\mathrm{up}}^{b} \\
\left(\mathrm{~cm}^{-1}\right)\end{array}$ & $P_{\mathrm{up}}^{c}$ & $J_{\text {up }}$ & $\log g f$ & $\begin{array}{c}g A \\
\left(\mathrm{~s}^{-1}\right)\end{array}$ & $\mathrm{CF}^{d}$ & $\log g f_{\mathrm{c}}^{\mathrm{e}}$ & $\begin{array}{l}g A_{\mathrm{c}}^{e} \\
\left(\mathrm{~s}^{-1}\right)\end{array}$ & $\begin{array}{l}\tau_{\mathrm{c}}^{f} \\
(\mathrm{~s})\end{array}$ & $\operatorname{Ref}^{g}$ \\
\hline 194.0573 & 24647 & (o) & 2 & 76178 & (e) & 3 & -2.03 & $1.66 \mathrm{E}+07$ & 0.030 & -1.67 & $3.78 \mathrm{E}+07$ & $3.76 \mathrm{E}-09$ & $\mathrm{~T}$ \\
\hline 200.1937 & 24647 & (o) & 2 & 74583 & (e) & 2 & -2.70 & $3.34 \mathrm{E}+06$ & 0.056 & -2.53 & $4.91 \mathrm{E}+06$ & $4.30 \mathrm{E}-09$ & $\mathrm{~T}$ \\
\hline 201.0298 & 24647 & (o) & 2 & 74375 & (e) & 3 & -2.84 & $2.35 \mathrm{E}+06$ & 0.003 & -2.66 & $3.55 \mathrm{E}+06$ & $4.11 \mathrm{E}-09$ & $\mathrm{~T}$ \\
\hline 204.2193 & 27227 & (o) & 2 & 76178 & (e) & 3 & -1.54 & $4.63 \mathrm{E}+07$ & 0.077 & -1.18 & $1.05 \mathrm{E}+08$ & $3.76 \mathrm{E}-09$ & $\mathrm{~T}$ \\
\hline 207.2838 & 26147 & (o) & 2 & 74375 & (e) & 3 & -4.21 & $9.53 \mathrm{E}+04$ & 0.005 & -4.03 & $1.44 \mathrm{E}+05$ & $4.11 \mathrm{E}-9$ & $\mathrm{~T}$ \\
\hline 209.2081 & 28394 & (o) & 4 & 76178 & (e) & 3 & -1.84 & $2.24 \mathrm{E}+07$ & 0.075 & -1.48 & $5.09 \mathrm{E}+07$ & $3.76 \mathrm{E}-09$ & $\mathrm{~T}$ \\
\hline 210.6890 & 28730 & (o) & 2 & 76178 & (e) & 3 & -1.78 & $2.50 \mathrm{E}+07$ & 0.076 & -1.42 & $5.69 \mathrm{E}+07$ & $3.76 \mathrm{E}-09$ & $\mathrm{~T}$ \\
\hline 211.1017 & 27227 & (o) & 2 & 74583 & (e) & 2 & -1.13 & $1.11 \mathrm{E}+08$ & 0.229 & -0.96 & $1.63 \mathrm{E}+08$ & $4.30 \mathrm{E}-09$ & $\mathrm{~T}$ \\
\hline 212.0315 & 27227 & (o) & 2 & 74375 & (e) & 3 & -2.82 & $2.25 \mathrm{E}+06$ & 0.187 & -2.64 & $3.40 \mathrm{E}+06$ & $4.11 \mathrm{E}-09$ & $\mathrm{~T}$ \\
\hline 212.4011 & 27517 & (o) & 1 & 74583 & (e) & 2 & -3.50 & $4.76 \mathrm{E}+05$ & 0.001 & -3.33 & $6.99 \mathrm{E}+05$ & $4.30 \mathrm{E}-09$ & $\mathrm{~T}$ \\
\hline 217.4143 & 28394 & (o) & 4 & 74375 & (e) & 3 & -0.65 & $3.15 \mathrm{E}+08$ & 0.355 & -0.47 & $4.76 \mathrm{E}+08$ & $4.11 \mathrm{E}-09$ & $\mathrm{~T}$ \\
\hline 218.0221 & 28730 & (o) & 2 & 74583 & (e) & 2 & -1.93 & $1.65 \mathrm{E}+07$ & 0.282 & -1.76 & $2.42 \mathrm{E}+07$ & $4.30 \mathrm{E}-09$ & $\mathrm{~T}$ \\
\hline 219.0141 & 28730 & (o) & 2 & 74375 & (e) & 3 & -1.54 & $4.04 \mathrm{E}+07$ & 0.442 & -1.36 & $6.10 \mathrm{E}+07$ & $4.11 \mathrm{E}-09$ & $\mathrm{~T}$ \\
\hline 220.3480 & 29214 & (o) & 3 & 74583 & (e) & 2 & -2.81 & $2.13 \mathrm{E}+06$ & 0.037 & -2.64 & $3.13 \mathrm{E}+06$ & $4.30 \mathrm{E}-09$ & $\mathrm{~T}$ \\
\hline 221.3613 & 29214 & (o) & 3 & 74375 & (e) & 3 & -0.83 & $2.01 \mathrm{E}+08$ & 0.426 & -0.65 & $3.04 \mathrm{E}+08$ & $4.11 \mathrm{E}-09$ & $\mathrm{~T}$ \\
\hline 224.3034 & 0 & (e) & 0 & 44569 & (o) & 1 & 0.05 & $1.52 \mathrm{E}+09$ & 0.359 & 0.08 & $1.32 \mathrm{E}+09$ & $1.20 \mathrm{E}-09$ & B \\
\hline 227.7468 & 32283 & (o) & 2 & 76178 & (e) & 3 & -1.31 & $6.36 \mathrm{E}+07$ & 0.084 & -0.95 & $1.45 \mathrm{E}+08$ & $3.76 \mathrm{E}-09$ & $\mathrm{~T}$ \\
\hline 228.6136 & 840 & (e) & 1 & 44569 & (o) & 1 & -4.03 & $1.22 \mathrm{E}+05$ & 0.028 & -4.00 & $1.06 \mathrm{E}+05$ & $1.20 \mathrm{E}-09$ & B \\
\hline 229.6898 & 1045 & (e) & 2 & 44569 & (o) & 1 & -2.70 & $2.58 \mathrm{E}+06$ & 0.024 & -2.67 & $2.25 \mathrm{E}+06$ & $1.20 \mathrm{E}-09$ & B \\
\hline
\end{tabular}

Notes. ${ }^{a}$ Derived from the experimental energy levels in Nilsson et al. (1991). Wavelengths longer than $200 \mathrm{~nm}$ are given in air.

${ }^{b}$ Nilsson et al. (1991). Rounded to the last digit.

${ }^{c}(\mathrm{e})$ and (o) stand for even and odd respectively.

${ }^{d}$ Cancellation factor (CF) as defined in Cowan (1981). The transition probability for which the CF is less than 0.1 is affected by a strong cancellation effect and should be taken with caution.

${ }^{e}$ Normalized using the experimental lifetime reported in the 13th column from the reference reported in the 14th column.

${ }^{f}$ Experimental lifetime of the upper level used to normalize the oscillator strength and the transition probability given respectively in columns 11 and 12.

${ }^{g}$ Reference of the experimental lifetime used to normalize the oscillator strength and the transition probability given respectively in columns 11 and $12 . \mathrm{T}=$ this work; B = Biémont et al. (2011); W = Wännström et al. (1988); H = Hannaford et al. (1982).

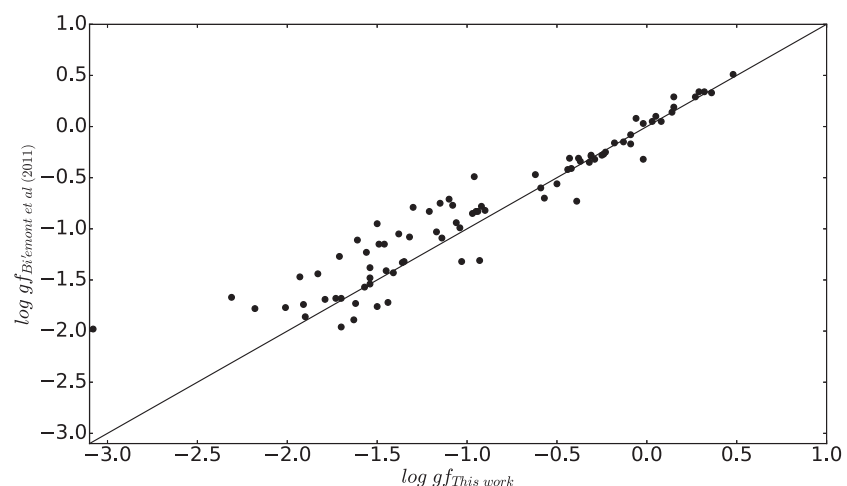

Figure 2. Comparison between the present HFR+CPOL $\log g f$ values and those of our previous study (Biémont et al. 2011). A straight line of equality has been drawn.

recommended as these values could be off by a few dex. Moreover, values of Kurucz (2011) that are weaker than ours by a few dex should be taken with care as we suspect that they are affected by strong cancellation effects similarly to the case of the line at $733.295 \mathrm{~nm}$ discussed previously.

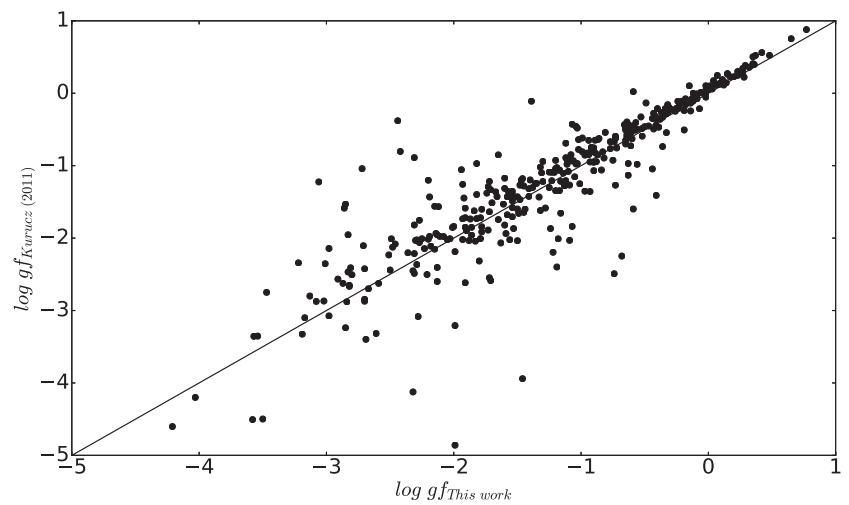

Figure 3. Comparison between the present HFR+CPOL $\log g f$ values and those of the Kurucz's data base (Kurucz 2011). A straight line of equality has been drawn.

In Fig. 4, it is seen that our HFR+CPOL $\log g f$-values agree well with the experimental determinations of Hannaford et al. (1982), the standard deviation of the differences between the two sets being 0.11 dex. From this comparison, one could estimate that the present 


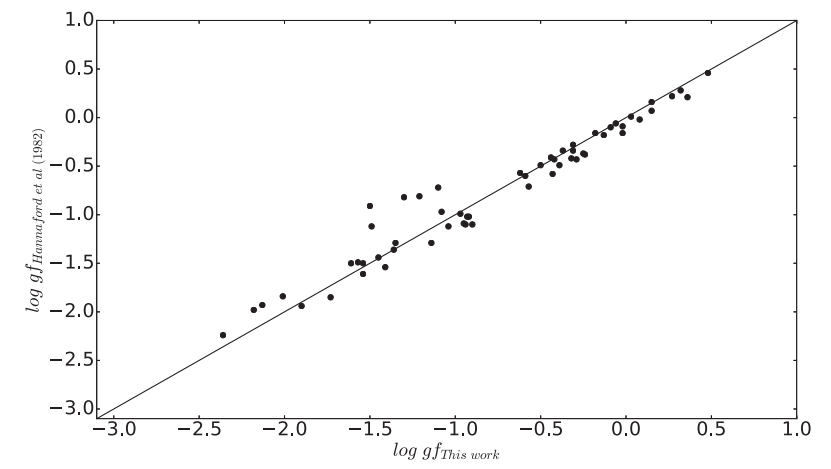

Figure 4. Comparison between the present HFR+CPOL $\log g f$ values and the experimental values of Hannaford et al. (1982). A straight line of equality has been drawn.

$\log g f$ values have an accuracy of the order of $\sim 0.1$ dex with the exception of the HFR+CPOL values affected by cancellation, i.e. with $\mathrm{CF}<0.1$.

\section{CONCLUSIONS}

New lifetimes have been measured for 22 highly excited evenparity levels in Y II using TR-LIF spectroscopy. A two-step laser excitation method has been used to reach these levels that belong to the configurations $4 d 6 s, 5 s 6 s 4 d 5 d, 5 p^{2}, 4 d 7 s$ and $4 d 6 d$. To reproduce our measurements, particularly for the levels belonging to the $4 \mathrm{~d} 7 \mathrm{~s}$ configuration, it was necessary to extend our previous HFR+CPOL model (Biémont et al. 2011) up to $n=10$. Comparisons of the present $\mathrm{HFR}+\mathrm{CPOL}$ calculations with previous and new measurements and theoretical data show a good agreement except for transitions affected by strong cancellations. In addition, it was found that the core-polarization effects in our model are slightly overestimated for the highly excited even-parity levels and consequently we choose to rescale our HFR + CPOL radiative rates using the experimental lifetimes for $357 \mathrm{E} 1$ transitions in $\mathrm{Y}$ II.

\section{ACKNOWLEDGEMENTS}

This work was financially supported by the Integrated Initiative of Infrastructure Project LASERLAB-EUROPE, contract LLC002268, by the Belgian F.R.S.-FNRS, and by the Swedish Research Council through the Linnaeus grant to the Lund Laser Centre, project grant 2011-4206 and the Knut and Alice Wallenberg Foundation. PQ and PP are, respectively, Research Director and Research Associate of the F.R.S.-FNRS. The Belgian team is grateful to the Swedish colleagues for the warm hospitality enjoyed at the Lund Laser Centre during the two campaigns in 2016 June and August.

\section{REFERENCES}

Andersen T., Ramanujan P. S., Bahr K., 1978, ApJ, 223, 344

Biémont É. et al., 2011, MNRAS, 414, 3350

Centre de Données astronomiques de Strasbourg (CDS), 2017, available at: http://cds.u-strasbg.fr

Cowan R. D., 1981, The Theory of Atomic Structure and Spectra. Univ. California Press, Berkeley

Gorshklov V. N., Komarovskii V. A., 1986, Opt. Spectrosc., 60, 541

Hannaford P., Lowe R. M., Grevesse N., Biémont É., Whaling W., 1982, ApJ, 261, 736

Johnsson W. R., Kolb D., Huang K.-N., 1983, At. Data Nucl. Data Tables, 28,333

Kurucz R. L., 2011, available at: http://kurucz.harvard.edu/atoms/3901

Lind K., Bergeman M., Asplund M., 2012, MNRAS, 427, 50

Lundberg H. et al., 2016, MNRAS, 460, 356

Migdalek J., Baylis W. E., 1987, Can. J. Phys., 65, 1612

Migdalek J., Stanek M., 1993, Z. Phys. D, 27, 9

Mishenina T. V., Korotin S. A., Carraro G., Kovtyukh V. V., Yegorova I. A., 2016, J. Phys. Conf. Ser., 665, 012025

Nilsson A. E., Johansson S., Kurucz R. L., 1991, Phys. Scr., 44, 226

Nissen P. E., 2015, A\&A, 579, A52

Palmeri P., Quinet P., Fivet V., Biémont É., Nilsson H., Engström L., Lundberg H., 2008, Physica Scripta, 78, 015304

Pirronello V., Strazzulla G., 1980, Ap\&SS, 72, 55

Pitts R. E., Newson G. H., 1986, J. Quant. Spectrosc. Radiat. Transfer, 35, 383

Quinet P., Palmeri P., Biémont É., McCurdy M. M., Rieger G., Pinnington E. H., Wickliffe M. E., Lawler J. E., 1999, MNRAS, 307, 934

Quinet P., Palmeri P., Biémont É., Li Z. S., Zhang Z. G., Svanberg S., 2002, J. Alloys Compd., 344, 255

Reshetnikova O. F., Skorokhod E. P., 1999, Opt. Spectrosc., 87, 829

Tucci Maia M., Ramírez I., Meléndez J., Bedell M., Bean J. L., Asplund M., 2016, A\&A, 590, A32

Wännström A., Vogel O., Arnesen A., Hallin R., 1988, Phys. Scr., 38, 564

\section{SUPPORTING INFORMATION}

Supplementary data are available at MNRAS online.

\section{etable4.txt}

Please note: Oxford University Press is not responsible for the content or functionality of any supporting materials supplied by the authors. Any queries (other than missing material) should be directed to the corresponding author for the article.

This paper has been typeset from a $\mathrm{T}_{\mathrm{E}} \mathrm{X} / \mathrm{LT}_{\mathrm{E}} \mathrm{X}$ file prepared by the author. 